IJMMS 26:11 (2001) 707-712

PII. S0161171201006299

http://ijmms.hindawi.com

(c) Hindawi Publishing Corp.

\title{
ON FUZZY POINTS IN SEMIGROUPS
}

\author{
KYUNG HO KIM
}

(Received 4 December 2000)

\begin{abstract}
We consider the semigroup $\underline{S}$ of the fuzzy points of a semigroup $S$, and discuss the relation between the fuzzy interior ideals and the subsets of $\underline{S}$ in an (intra-regular) semigroup $S$.
\end{abstract}

2000 Mathematics Subject Classification. 03E72, $20 \mathrm{M} 12$.

1. Introduction. After the introduction of the concept of fuzzy sets by Zadeh [8], several researches were conducted on the generalizations of the notion of fuzzy sets. Pu and Liu [5] introduced the notion of fuzzy points. In [6, 7, 8], authors characterized fuzzy ideals as fuzzy points of semigroups. In [1, 2, 3], Kuroki discussed the properties of fuzzy ideals and fuzzy bi-ideals in a semigroup and a regular semigroup. In this paper, we consider the semigroup $\underline{S}$ of the fuzzy points of a semigroup $S$, and discuss the relation between the fuzzy interior ideals and the subsets of $\underline{S}$ in an (intra-regular) semigroup $S$.

2. Preliminaries. Let $S$ be a semigroup with a binary operation ".". A nonempty subset $A$ of $S$ is called a subsemigroup of $S$ if $A^{2} \subseteq A$, a left (resp., right) ideal of $S$ if $S A \subseteq A$ (resp., $A S \subseteq A$ ), and a two-sided ideal (or simply ideal) of $S$ if $A$ is both a left and a right ideal of $S$. A subsemigroup $A$ of $S$ is called a bi-ideal of $S$ if $A S A \subseteq A$. Let $S$ be a semigroup. A nonempty subset $A$ of $S$ is called an interior ideal of $S$ if $S A S \subseteq A$. A function $f$ from a set $X$ to $[0,1]$ is called a fuzzy subset of $X$. The set $\{x \in X \mid$ $f(x)>0\}$ is called the support, denoted by $\operatorname{supp} f$, of $f$. The closed interval $[0,1]$ is a complete lattice with two binary operations " $\vee$ " and “ $\wedge$ ", where $\alpha \vee \beta=\sup \{\alpha, \beta\}$ and $\alpha \wedge \beta=\inf \{\alpha, \beta\}$ for each $\alpha, \beta \in[0,1]$. For any $\alpha \in(0,1]$ and $x \in X$, a fuzzy subset $x_{\alpha}$ of $X$ is called a fuzzy point in $X$ if

$$
x_{\alpha}(y)= \begin{cases}\alpha & \text { if } x=y \\ 0 & \text { otherwise }\end{cases}
$$

for each $y \in X$. If $f$ is a fuzzy subset of $X$, then a fuzzy point $x_{\alpha}$ is said to be contained in $f$, denoted by $x_{\alpha} \in f$, if $\alpha \leq f(x)$. It is clear that $x_{\alpha} \in f$ for some $\alpha \in(0,1]$ if and only if $x \in \operatorname{supp} f$.

A fuzzy subset $f$ of a semigroup $S$ is called a fuzzy subsemigroup of $S$ if

$$
f(x y) \geq f(x) \wedge f(y),
$$

for all $x, y \in S$, a fuzzy left (resp., right) ideal of $S$ if 


$$
f(x y) \geq f(y) \text { (resp., } f(x y) \geq f(x)),
$$

for all $x, y \in S$, and a fuzzy ideal of $S$ if $f$ is both a fuzzy left and a fuzzy right ideal of $S$. It is clear that $f$ is a fuzzy ideal of a semigroup $S$ if and only if $f(x y) \geq$ $f(x) \vee f(y)$ for all $x, y \in S$, and that every fuzzy left (right, two-sided) ideal of $S$ is a fuzzy subsemigroup of $S$.

3. Interior ideals of fuzzy points. Let $\mathscr{F}(S)$ be the set of all fuzzy subsets of a semigroup $S$. For each $f, g \in \mathscr{F}(S)$, the product of $f$ and $g$ is a fuzzy subset $f \circ g$ defined as follows:

$$
(f \circ g)(x)= \begin{cases}\bigvee(f(y) \wedge g(z)) & \text { if } x=y z(y, z \in S), \\ 0 & \text { otherwise, }\end{cases}
$$

for each $x \in S$. It is clear that $(f \circ g) \circ h=f \circ(g \circ h)$, and that if $f \subseteq g$, then $f \circ h \subseteq g \circ h$ and $h \circ f \subseteq h \circ g$ for any $f, g$, and $h \in \mathscr{F}(S)$. Thus $\mathscr{F}(S)$ is a semigroup with the product "o".

Let $\underline{S}$ be the set of all fuzzy points in a semigroup $S$. Then $x_{\alpha} \circ y_{\beta}=(x y)_{\alpha \wedge \beta} \in \underline{S}$ and $x_{\alpha} \circ\left(y_{\beta} \circ z_{\gamma}\right)=(x y z)_{\alpha \wedge \beta \wedge \gamma}=\left(x_{\alpha} \circ y_{\beta}\right) \circ z_{\gamma}$ for any $x_{\alpha}, y_{\beta}$, and $z_{\gamma} \in \underline{S}$. Thus $\underline{S}$ is a subsemigroup of $\mathscr{F}(S)$.

For any $f \in \mathscr{F}(S), f$ denotes the set of all fuzzy points contained in $f$, that is, $\underline{f}=\left\{x_{\alpha} \in \underline{S} \mid f(x) \geq \bar{\alpha}\right.$. If $x_{\alpha} \in \underline{S}$, then $\alpha>0$.

For any $A, B \subseteq \underline{S}$, we define the product of two sets $A$ and $B$ as $A \circ B=\left\{x_{\alpha} \circ y_{\beta} \mid\right.$ $\left.x_{\alpha} \in A, y_{\beta} \in B\right\}$.

LEMMA 3.1 (see [7, Lemma 4.1]). Let $f$ be a nonzero fuzzy subset of a semigroup $S$. Then the following conditions are equivalent:

(1) $f$ is a fuzzy left (right, two-sided) ideal of $S$.

(2) $\underline{f}$ is a left (right, two-sided) ideal of $\underline{S}$.

LEMMA 3.2 (see [7, Lemma 4.2]). Let $f$ and $g$ be two fuzzy subsets of a semigroup $S$. Then

(1) $\underline{f \cup g}=\underline{f \cup g}$.

(2) $\overline{f \cap g}=\bar{f} \cap \bar{g}$.

(3) $\underline{\underline{f \circ g}} \supseteq \underline{f} \circ \underline{g}$.

A fuzzy subsemigroup $f$ of a semigroup $S$ is called a fuzzy interior ideal of $S$ if $f(x a y) \geq f(a)$ for all $x, a, y \in S$.

LEMMA 3.3. Let $f$ be a nonzero fuzzy subset of a semigroup $S$. Then the following conditions are equivalent:

(1) $f$ is a fuzzy interior ideal of $S$.

(2) $\underline{f}$ is an interior ideal of $\underline{S}$.

Proof. Let $f$ be a fuzzy interior ideal of $S$, and let $x_{\alpha}, z_{\gamma} \in \underline{S}$ and $y_{\beta} \in \underline{f}$. Then since $\alpha>0, \gamma>0$, and $0<\beta \leq f(y)$, we have

$$
0<\alpha \wedge \beta \wedge \gamma \leq \alpha \wedge f(y) \wedge \gamma \leq f(y) \leq f(x y z)
$$


Hence $x_{\alpha} \circ y_{\beta} \circ z_{\gamma}=(x y z)_{\alpha \wedge \beta \wedge \gamma} \in \underline{f}$. This implies that $\underline{S} \circ \underline{f} \circ \underline{S} \subseteq \underline{f}$, thus $\underline{f}$ is an interior ideal of $\underline{S}$. Conversely, suppose that $f$ is an interior ideal of $\underline{S}$. Let $x, y, z \in S$. If $f(y)=0$, then $f(y)=0 \leq f(x y z)$. If $f(y) \neq 0$, then $y_{f(y)} \in \underline{f}$ and $x_{f(y)}, z_{f(y)} \in \underline{S}$. Since $\underline{f}$ is an interior ideal of $\underline{S}$, we have

$$
(x y z)_{f(y)}=(x y z)_{f(y) \wedge f(y) \wedge f(y)}=x_{f(y)} \circ y_{f(y)} \circ z_{f(y)} \in \underline{f} .
$$

This implies that $f(x y z) \geq f(y)$, and hence $f$ is a fuzzy interior ideal of $S$.

It is clear that any ideal of a semigroup $S$ is an interior ideal of $S$. It is also clear that any fuzzy ideal of $S$ is a fuzzy interior ideal of $S$. A semigroup $S$ is called regular if, for each element $a$ of $S$, there exists an element $x$ in $S$ such that $a=a x a$.

THEOREM 3.4. Let $f$ be any fuzzy set in a regular semigroup $S$. Then the following conditions are equivalent:

(1) $f$ is a fuzzy right (resp., left) ideal of $S$.

(2) $\underline{f}$ is an interior ideal of $\underline{S}$.

Proof. It suffices to show that (2) implies (1). Assume that (2) holds. Let $x$ be any element in $S$. Then since $S$ is regular, there exists element $a$ in $S$ such that $x=x a x$. If $f(x)=0, f(x)=0 \leq f(x y)$. If $f(x) \neq 0$, then $x_{f(x)} \in \underline{f}$ and $y_{f(x)} \in \underline{S}$. Since $\underline{f}$ is an interior ideal of $\underline{S}$, we have

$$
\begin{aligned}
(x y)_{f(x)} & =(x a x y)_{f(x)} \\
& =((x a) x y)_{f(x) \wedge f(x) \wedge f(x)} \\
& =(x a)_{f(x)} \circ x_{f(x)} \circ y_{f}(x) \in \underline{f .}
\end{aligned}
$$

This implies that $f(x y) \geq f(x)$, and hence $f$ is a fuzzy right ideal of $S$.

THEOrem 3.5 (see [7, Theorem 3.3]). Let $S$ be a semigroup. If for a fixed $\alpha \in(0,1]$, $f_{\alpha}: S \rightarrow \underline{S}$ is a function defined by $f_{\alpha}(x)=x_{\alpha}$, then $f_{\alpha}$ is a one-to-one homomorphism of semigroups.

From Theorem 3.5, we can consider $\underline{S}$ as an extension of a semigroup $S$.

Let $f$ be a fuzzy subset of a semigroup $S$. If $\mathscr{R}_{f}$ is the subset of $\underline{S} \times \underline{S}$ given as following:

$$
\mathscr{R}_{f}=\left\{\left(x_{\alpha}, x_{\alpha}\right) \mid x_{\alpha} \notin \underline{f}\right\} \cup\left\{\left(x_{\alpha}, x_{\beta}\right) \mid x_{\alpha}, x_{\beta} \in \underline{f}\right\},
$$

then the set $\mathscr{R}_{f}$ is an equivalence relation on $\underline{S}$. We can consider the quotient set $\underline{S} / \mathscr{R}_{f}$, with the equivalence classes $\bar{x}_{\alpha}$ for each $x \in S$. We will denote the subset $\left\{\bar{x}_{\alpha} \mid x_{\alpha} \in \underline{f}\right\}$ of $\underline{S} / \Re_{f}$ by $E(\underline{f})$. If $\bar{x}_{\alpha} \in E(\underline{f})$, then $\bar{x}_{\alpha}=\bar{x}_{f(x)}=\left\{x_{\lambda} \mid 0<\lambda \leq f(x)\right\}$. If $\bar{x}_{\alpha} \notin E(\underline{f})$, then $\bar{x}_{\alpha}=\left\{x_{\alpha}\right\}$ (singleton set).

Let $f$ be a fuzzy subsemigroup of $S$. If the product “*” on $E(\underline{f})$ is defined by $\bar{x}_{\alpha} * \bar{y}_{\beta}=\overline{(x y)}_{\alpha \wedge \beta}$ for each $\bar{x}_{\alpha}, \bar{y}_{\beta} \in E(\underline{f})$, then $E(\underline{f})$ is a semigroup under the operation “*”.

THEOREM 3.6. Let $f$ be a fuzzy interior ideal of $S$. Then $E(\underline{f})$ is an interior ideal of $\left(\underline{S} / \mathscr{R}_{f}, *\right)$. 
Proof. Let $\bar{x}_{\alpha}, \bar{y}_{\beta} \in \underline{S} / \mathscr{R}_{f}$ and $\bar{a}_{\gamma} \in E(\underline{f})$. Then since $x_{\alpha}, y_{\beta} \in \underline{S}, a_{\gamma} \in \underline{f}$ and $\underline{f}$ is an interior ideal of $\underline{S},(x a y)_{\alpha \wedge \gamma \wedge \beta}=x_{\alpha} \circ a_{\gamma} \circ \bar{y}_{\beta} \in \underline{f}$. Hence $\bar{x}_{\alpha} * \bar{a}_{\gamma} * \bar{y}_{\beta}=\overline{(x a y)}_{\alpha \wedge \gamma \wedge \beta} \in$ $E(\underline{f})$. It follows that $E(\underline{f})$ is an interior ideal of $\underline{S} / \Re_{f}$.

A semigroup $S$ is called intra-regular if, for each element $a$ of $S$, there exists elements $x$ and $y$ in $S$ such that $a=x a^{2} y$.

THEOREM 3.7. A semigroup $S$ is intra-regular if and only if the semigroup $\underline{S}$ is intraregular.

Proof. Let $a_{\alpha} \in \underline{S}$. Then since $S$ is intra-regular and $a \in S$, there exist $x, y$ in $S$ such that $a=x a^{2} y$. Thus $x_{\alpha} \in \underline{S}$ and $y_{\alpha} \in \underline{S}$ and

$$
x_{\alpha} \circ a_{\alpha} \circ a_{\alpha} \circ y_{\alpha}=x_{\alpha} \circ\left(a^{2}\right)_{\alpha} \circ y_{\alpha}=\left(x a^{2} y\right)_{\alpha}=a_{\alpha} .
$$

Hence $\underline{S}$ is intra-regular. Conversely, let $\underline{S}$ be intra-regular and $a \in S$. Then for any $\alpha \in(0,1]$, there exist elements $x_{\beta}, y_{\delta} \in \underline{S}$ such that

$$
a_{\alpha}=x_{\beta} \circ a_{\alpha} \circ a_{\alpha} \circ y_{\delta}=\left(x a^{2} y\right)_{\beta \wedge \alpha \wedge \delta} .
$$

This implies that $a=x a^{2} y$ and $x, y \in S$.

THEOREM 3.8. For a fuzzy set $f$ of an intra-regular semigroup $S$ the following conditions are equivalent:

(1) $f$ is a right (resp., left) ideal of $S$.

(2) $\underline{f}$ is an interior ideal of $\underline{S}$.

Proof. It is clear that (1) implies (2). Assume that (2) holds. Let $x, y$ be any elements in $S$. Then since $S$ is intra-regular, there exist elements $a, b$ in $S$ such that $x=a x^{2} b$. If $f(x)=0, f(x)=0 \leq f(x y)$. If $f(x) \neq 0$, then $x_{f(x)} \in \underline{f}$ and $y_{f(x)} \in \underline{S}$. Since $\underline{f}$ is an interior ideal of $\underline{S}$, we have

$$
\begin{aligned}
(x y)_{f(x)} & =\left(a x^{2} b y\right)_{f(x)} \\
& =((a x) x(b y))_{f(x) \wedge f(x) \wedge f(x)} \\
& =(a x)_{f(x)} \circ x_{f(x)} \circ(b y)_{f}(x) \in \underline{f .}
\end{aligned}
$$

This implies that $f(x y) \geq f(x)$, and hence $f$ is a fuzzy right ideal of $S$.

LEMMA 3.9 (see [3, Lemma 4.1]). For a semigroup $S$, the following conditions are equivalent:

(1) $S$ is intra-regular.

(2) $L \cap R \subset L R$ holds for every left ideal $L$ and right ideal $R$ of $S$.

LEMMA 3.10 (see [3, Lemma 4.2]). For a semigroup $S$, the following conditions are equivalent:

(1) $S$ is intra-regular.

(2) $f \cap g \subset g \circ f$ holds for every fuzzy right ideal $f$ and fuzzy left ideal $g$ of $S$.

THEOREM 3.11. For a semigroup $S$, the following conditions are equivalent:

(1) $S$ is intra-regular.

(2) $\underline{f} \cap \underline{g} \subset \underline{g} \circ \underline{f}$ for every fuzzy right ideal $f$ and every fuzzy left ideal $g$ of $S$. 
Proof. Let $f$ be a fuzzy right ideal and $g$ a left ideal of $S$. Since $\underline{S}$ is intra-regular, $f$ is a right ideal, and $\underline{g}$ is a left ideal of $\underline{S}, f \cap \underline{g} \subset \underline{g} \circ f$ by Lemma 3.9.

Conversely, let $f$ be a fuzzy right ideal and $g$ a fuzzy left ideal of $S$. Let $x \in S$. If $f(x)=0$ or $g(x)=0$, then

$$
0=f(x) \wedge g(x) \subseteq(g \circ f)(x) .
$$

If $f(x) \neq 0$ and $g(x) \neq 0$, then $x_{f(x) \wedge g(x)} \in \underline{f}$ and $x_{f(x) \wedge g(x)} \in \underline{g}$. Hence

$$
x_{f(x) \wedge g(x)} \in \underline{f} \cap \underline{g} \subset \underline{g} \circ \underline{f} \subseteq \underline{g} \circ \underline{f} .
$$

It follows that $f(x) \wedge g(x) \subseteq(g \circ f)(x)$. Hence $(f \cap g)(x)=f(x) \wedge g(x) \subseteq(g \circ f)(x)$ for all $x \in S$ and $f \cap g \subset g \circ f$. By Lemma 3.10, $S$ is intra-regular.

LEMMA 3.12 (see [4, Lemma 4.3]). For a semigroup $S$ the following conditions are equivalent:

(1) $S$ is both regular and intra-regular.

(2) $B^{2}=B$ for every bi-ideal $B$ of $S$.

(3) $A \cap B \subset A B \cap B A$ for all bi-ideals $A$ and $B$ of $S$.

(4) $B \cap L \subset B L \cap L B$ for every bi-ideal $B$ and every left ideal $L$ of $S$.

(5) $B \cap R \subset B R \cap R B$ for every bi-ideal $B$ and every right ideal $R$ of $S$.

(6) $L \cap R \subset L R \cap R L$ for every right ideal $R$ and every left ideal $L$ of $S$.

A fuzzy subsemigroup $f$ of $S$ is called a fuzzy bi-ideal of $S$ if $f(x y z) \geq f(x) \wedge f(z)$ for all $x, y$ and $z \in S$.

COROLLARY 3.13. For a semigroup $S$ the following conditions are equivalent:

(1) $S$ is both regular and intra-regular.

(2) $\underline{f} \circ \underline{f}=\underline{f}$ for every fuzzy bi-ideal $f$ of $S$.

(3) $\bar{f} \cap \underline{g} \subset \underline{f} \circ \underline{g} \cap \underline{g} \circ \underline{f}$ for all fuzzy bi-ideals $f$ and $g$ of $S$.

(4) $\underline{f} \cap \underline{g} \subset \underline{f} \circ \underline{g} \cap \underline{g} \circ \underline{f}$ for every fuzzy bi-ideal $f$ and every fuzzy left ideal $g$ of $S$.

(5) $\bar{f} \cap \underline{\bar{g}} \subset \bar{f} \circ \underline{\bar{g}} \cap \underline{\bar{g}} \circ \underline{f}$ for every fuzzy bi-ideal $f$ and every fuzzy right ideal $g$ of $S$.

(6) $\bar{f} \cap \underline{\bar{g}} \subset \bar{f} \circ \underline{\bar{g}} \cap \underline{g} \circ \underline{f}$ for every fuzzy right ideal $f$ and every fuzzy left ideal $g$ of $S$.

\section{REFERENCES}

[1] N. Kuroki, On fuzzy ideals and fuzzy bi-ideals in semigroups, Fuzzy Sets and Systems 5 (1981), no. 2, 203-215. MR 82e:20076. Zbl 452.20060.

[2] _ _ Fuzzy semiprime ideals in semigroups, Fuzzy Sets and Systems 8 (1982), no. 1, 71-79. MR 83h:20073. Zbl 488.20049.

[3] _ On fuzzy semigroups, Inform. Sci. 53 (1991), no. 3, 203-236. MR 91j:20144. Zbl 714.20052.

[4] S. Lajos, Theorems on $(1,1)$-ideals in Semigroups. II, Department of Mathematics, Karl Marx University for Economics, Budapest, 1974. MR 51\#3333. Zbl 291.20074.

[5] P. M. Pu and Y. M. Liu, Fuzzy topology. I. Neighborhood structure of a fuzzy point and MooreSmith convergence, J. Math. Anal. Appl. 76 (1980), no. 2, 571-599. MR 82e:54009a. Zbl 447.54006 .

[6] X. P. Wang, Z. W. Mo, and W. J. Liu, Fuzzy ideals generated by fuzzy point in semigroups, Sichuan Shifan Daxue Xuebao Ziran Kexue Ban 15 (1992), no. 4, 17-24. MR 94b:20067.

[7] Y. H. Yon, The semigroups of fuzzy points, submitted in Comm. Algebra. 
[8] L. A. Zadeh, Fuzzy sets, Information and Control 8 (1965), 338-353. MR 36\#2509. Zbl 139.24606.

Kyung Ho Kim: Department of Mathematics, ChunguU National UniVersity, ChungJu 380-702, KOREA

E-mail address: ghkim@gukwon.chungju.ac.kr 




Advances in

Operations Research

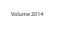

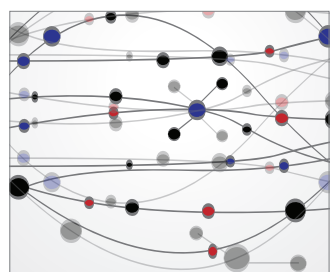

\section{The Scientific} World Journal
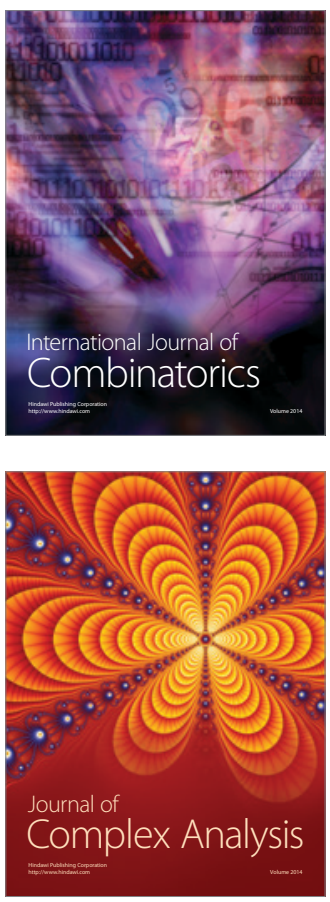

International Journal of

Mathematics and

Mathematical

Sciences


Journal of

Applied Mathematics


Submit your manuscripts at http://www.hindawi.com
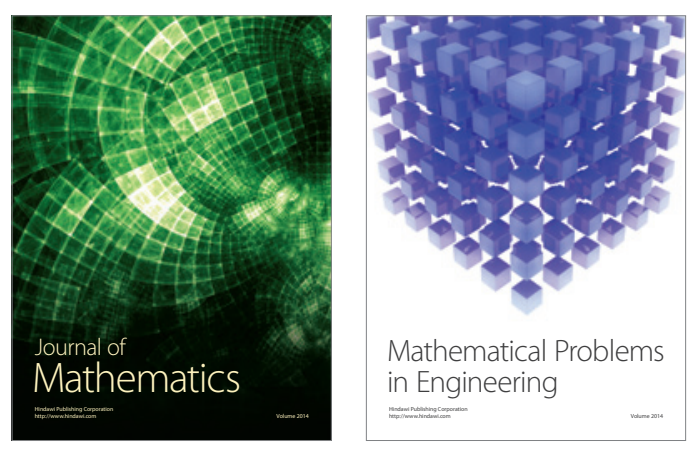

Mathematical Problems in Engineering
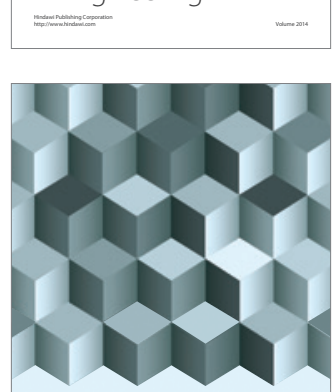

Journal of

Function Spaces
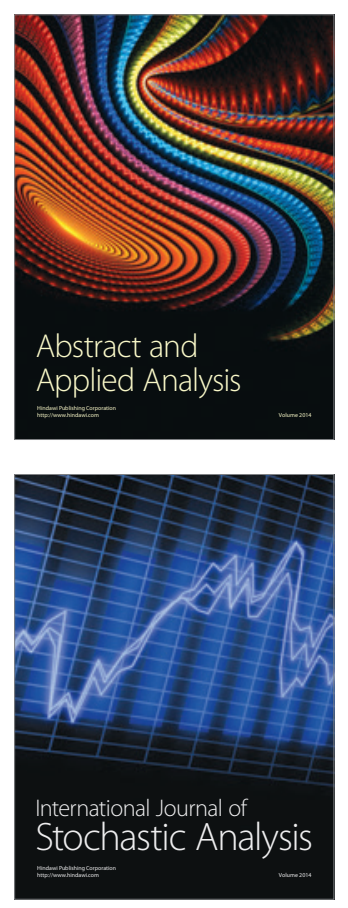

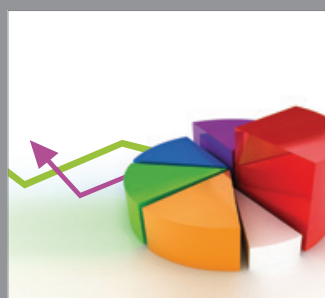

ournal of

Probability and Statistics

Promensencen
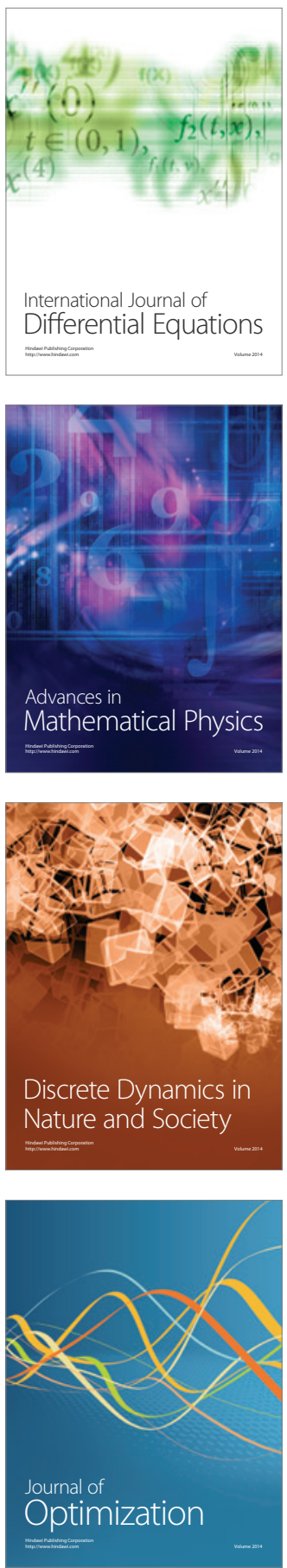